\title{
Brain metabolic changes in Hodgkin disease patients following diagnosis and during the disease course: An ${ }^{18}$ F-FDG PET/CT study
}

\author{
AGOSTINO CHIARAVALLOTI $^{1}$, MARCO PAGANI ${ }^{2,3}$, MARIA CANTONETTI ${ }^{1}$, BARBARA DI PIETRO $^{1}$, \\ MARIO TAVOLOZZA ${ }^{1}$, LAURA TRAVASCIO ${ }^{1}$, DANIELE DI BIAGIO ${ }^{1}$, \\ ROBERTA DANIELI $^{1}$ and ORAZIO SCHILLACI ${ }^{1,4}$ \\ ${ }^{1}$ Department of Biomedicine and Prevention, University of Rome Tor Vergata, Rome I-00133; \\ ${ }^{2}$ Institute of Cognitive Sciences and Technologies, The National Research Council, Rome I-00185, Italy; \\ ${ }^{3}$ Department of Nuclear Medicine, Karolinska Hospital, Stockholm SE-17176, Sweden; \\ ${ }^{4}$ Department of Nuclear Medicine, IRCCS Neuromed, Pozzilli I-86077, Italy
}

Received March 2, 2014; Accepted October 15, 2014

DOI: $10.3892 / \mathrm{ol} .2014 .2765$

\begin{abstract}
The aim of the present study was to investigate brain glucose metabolism in patients with Hodgkin disease (HD) after diagnosis and during chemotherapy treatment. Following the administration of first-line doxorubicin, bleomycin, vinblastine and dacarbazine (ABVD) chemotherapy, 74 HD patients underwent ${ }^{18} \mathrm{~F}$-fluoro-2-deoxy-D-glucose $\left({ }^{18} \mathrm{~F}\right.$-FDG) positron emission tomography (PET)/computed tomography brain scans, both baseline (PET0) and interim (PET2) at the Department of Biomedicine and Prevention, University of Rome Tor Vergata (Rome, Italy). Fifty-seven patients were further evaluated $15 \pm 6$ days after four additional cycles (PET6). Furthermore, a control group (CG) of 40 chemotherapy-naïve subjects was enrolled. Differences in brain ${ }^{18} \mathrm{~F}$-FDG uptake between the $\mathrm{CG}$, PET0, PET2 and PET6 scans were analyzed using statistical parametric mapping. Compared with the PET0 and CG scans, the PET2 scan demonstrated a higher metabolic activity in Brodmann area (BA) 39, and a metabolic reduction in BA 11 bilaterally and in left BA 32 . All of these changes disappeared at PET6. The results of the present study indicate that ABVD chemotherapy has a limited impact on brain metabolism.
\end{abstract}

\section{Introduction}

Hodgkin disease (HD) is a lymphoproliferative disorder with an incidence rate of $2.7-2.8$ per 100,000 individuals in the UK and the USA in 2013 (1). Doxorubicin, bleomycin,

Correspondence to: Miss. Agostino Chiaravalloti, Department of Biomedicine and Prevention, University of Rome Tor Vergata, 81 Viale Oxford, Rome I-00133, Italy

E-mail: agostino.chiaravalloti@gmail.com

Key words: adriamycin, bleomycin, vinblastine and dacarbazine, chemotherapy, chemobrain, Hodgkin disease, positron emission tomography vinblastine and dacarbazine (ABVD) chemotherapy (CHT) is routinely used in the treatment of $\mathrm{HD}(2)$, and is less toxic than other chemotherapeutic schemes, such as mechlorethamine, vincristine, procarbazine and prednisone (3), and bleomycin, etoposide, doxorubicin, cyclophosphamide, vincristine, procarbazine and prednisone regimens (4).

In a recent study investigating the brain glucose metabolism in HD patients by means of $2-\left[{ }^{18} \mathrm{~F}\right]$ fluoro-2-deoxy-D-glucose $\left({ }^{18} \mathrm{~F}-\mathrm{FDG}\right)$ positron emission tomography/computed tomography (PET/CT), the hypothesis of brain damage induced by ABVD CHT treatment was dismissed (5). However, a strong body of evidence demonstrates the coexistence of depression and cancer, with a $20-50 \%$ prevalence of depression in patients exhibiting solid tumors (6). Furthermore, various studies report a direct correlation between the rapid progression of cancer and severe depression (7). This is consistent with our previous study, which supports the hypothesis that metabolic changes following CHT may correlate with the transient depressive state of cancer patients after diagnosis, followed by an improvement in general and psychological conditions due to a positive therapeutic response (5).

The aim of the present study was to investigate the impact of ABVD CHT on a larger cohort of HD patients, evaluating the brain metabolic changes during the various steps of CHT and comparing them with an age-matched control group (CG). Furthermore, the possible role of disease severity as a variable affecting the brain metabolic changes was investigated in the HD patients.

\section{Patients and methods}

Patients and CG. From September 2008 to September 2012, 74 patients (males, 32; females, 42; mean age, $32 \pm 11$ years) with biopsy-diagnosed HD, who were included in a national study evaluating the early treatment response to ABVD CHT (8), underwent a ${ }^{18} \mathrm{~F}-\mathrm{FDG}$ PET/CT brain scan in 3D mode (9) in association with a whole body staging PET/CT study. According to the Ann Arbor staging Criteria (10), 11, 
Table I. Statistical parametric mapping comparisons between ${ }^{18} \mathrm{~F}-\mathrm{FDG}$ uptake in PET2 and CG.

\begin{tabular}{|c|c|c|c|c|c|c|c|}
\hline \multirow[b]{2}{*}{ Comparison } & \multicolumn{3}{|c|}{ Cluster level } & \multicolumn{4}{|c|}{ Voxel level } \\
\hline & $\begin{array}{l}\text { Cluster } \\
\text { extent }\end{array}$ & $\begin{array}{c}\text { Corrected } \\
\text { P-value }\end{array}$ & $\begin{array}{c}\text { Cortical } \\
\text { lobe }\end{array}$ & $\begin{array}{l}\text { Talairach } \\
\text { coordinates }\end{array}$ & $\begin{array}{l}\text { Maximum } \\
\text { Z score }\end{array}$ & $\begin{array}{l}\text { Cortical } \\
\text { region }\end{array}$ & BA \\
\hline \multirow[t]{2}{*}{ CG-PET2 } & 1729 & 0.036 & L limbic & $-4,43,7$ & 4.16 & Anterior cingulate cortex & 32 \\
\hline & & & L frontal & $-13,48,22$ & 3.29 & Superior frontal gyrus & 11 \\
\hline PET2-CG & 546 & 0.048 & $\mathrm{R}$ parietal & $48,-71,33$ & 4.78 & Angular gyrus & 39 \\
\hline
\end{tabular}

For each significant cluster, the number of voxels, the corrected P-value and the cortical lobe where the voxel is located are reported at the cluster level; and the coordinates of the Talairach correlation sites, the Z-score of the maximum correlation point, and the corresponding cortical region and BA are reported at the voxel level. When the maximum correlation is achieved outside of the gray matter, the gray matter (BA) in the closest proximity was identified (range, $0-3 \mathrm{~mm}$ ). Thresholds of $\mathrm{P}<0.05$, corrected for multiple comparisons using the false discovery rate, and $\mathrm{P}<0.001$ uncorrected at voxel level, were implemented for PET2 and CG comparisons.. L, left; R, right; BA, Brodmann's area.

Table II. Statistical parametric mapping comparisons between ${ }^{18} \mathrm{~F}-\mathrm{FDG}$ uptake in PET0 and PET2.

\begin{tabular}{|c|c|c|c|c|c|c|c|}
\hline \multirow[b]{2}{*}{ Comparison } & \multicolumn{3}{|c|}{ Cluster level } & \multicolumn{4}{|c|}{ Voxel level } \\
\hline & $\begin{array}{l}\text { Cluster } \\
\text { extent }\end{array}$ & $\begin{array}{l}\text { Corrected } \\
\text { P-value }\end{array}$ & $\begin{array}{l}\text { Cortical } \\
\text { lobe }\end{array}$ & $\begin{array}{l}\text { Talairach } \\
\text { coordinates }\end{array}$ & $\begin{array}{l}\text { Maximum } \\
\text { Z score }\end{array}$ & $\begin{array}{l}\text { Cortical } \\
\text { region }\end{array}$ & BA \\
\hline \multirow[t]{4}{*}{ PET0-PET2 } & 1450 & $0.017^{\mathrm{a}}$ & L limbic & $-4,44,6$ & 4.21 & Anterior cingulate cortex & 32 \\
\hline & & & $\mathrm{R}$ frontal & $4,52,-12$ & 3.64 & Medial frontal gyrus & 11 \\
\hline & & & L frontal & $-8,48,-26$ & 3.49 & Orbital gyrus & 11 \\
\hline & & & L frontal & $-14,48,-24$ & 3.47 & Superior frontal gyrus & 11 \\
\hline PET2-PET0 & 844 & $0.042^{\mathrm{a}}$ & $\mathrm{R}$ parietal & $48,-72,34$ & 4.55 & Angular gyrus & 39 \\
\hline
\end{tabular}

For each significant cluster, the number of voxels, the corrected P-value and the cortical lobe where the voxel is located are reported at the cluster level; and the coordinates of the Talairach correlation sites, the Z-score of the maximum correlation point, and the corresponding cortical region and BA are reported at the voxel level. When the maximum correlation is achieved outside of the gray matter, the gray matter (BA) in the closest proximity was identified (range, 0-3 mm). Thresholds of $\mathrm{P}<0.05$, corrected for multiple comparisons using the false discovery rate, and $\mathrm{P}<0.001$ uncorrected at voxel level, were implemented for PET0-PET2 and PET2-PET0 comparisons. ${ }^{\text {a }}<0.05$ corrected for multiple comparisons at cluster level. ${ }^{18} \mathrm{~F}-\mathrm{FDG}, 2$-[18F] fluoro-2-deoxy-D-glucose; PET, positron emission tomography; CT, computed tomography; PET0, brain ${ }^{18} \mathrm{~F}$-FDG PET/CT scan within one week of Hodgkin disease diagnosis; PET2, brain ${ }^{18} \mathrm{~F}-\mathrm{FDG}$ PET/CT scan $15 \pm 5$ days after the first two adriamycin, bleomycin, vinblastine and dacarbazine cycles; CG, control group; BA, Brodmann area; L, left; R, right.

35, 11 and 17 patients exhibited stages I, II, III and IV HD, respectively.

In accordance with previous studies (11-13), all patients underwent the initial PET/CT scan (PET0) within one week of HD diagnosis. A second PET/CT scan (PET2) was performed in all patients, $15 \pm 5$ days after the first two ABVD cycles. Written informed consent was obtained for a third brain PET/CT scan (PET6) from 57 patients (females, 31; males, 26; mean age, $31 \pm 10$ years), which was performed $15 \pm 4$ days after four additional one-month ABVD cycles, for a total of six cycles.

Forty CHT-naïve subjects (males, 22; females, 18; mean age, $36 \pm 7$ years) undergoing a ${ }^{18} \mathrm{~F}-\mathrm{FDG} \mathrm{PET} / \mathrm{CT}$ and found to be completely negative for various diseases were enrolled in the study and served as the CG (14).

Patients and CG subjects with a history of diabetes, other carcinomas, human immunodeficiency virus, neurological, psychiatric or mood disorders, surgery, radiation or trauma to the brain were excluded from the present study. Furthermore, a number of patients treated with agents that could interfere with
${ }^{18} \mathrm{~F}-\mathrm{FDG}$ uptake and distribution in the brain were excluded from the present study (16). No patients demonstrated liver or renal damage, and no patients were pregnant or breastfeeding. Informed consent was obtained from all of the patients, in accordance with the Declaration of Helsinki, and the present study was approved by the ethics committee of the Hospital of Tor Vergata (Rome, Italy).

Treatment strategy. ABVD cycles were repeated every 28 days. A cycle of treatment consisted of the following on day one: doxorubicin, $25 \mathrm{mg} / \mathrm{m}^{2}$; bleomycin, 10,000 units $/ \mathrm{m}^{2}$; vinblastine, $6 \mathrm{mg} / \mathrm{m}^{2}$; and dacarbazine, $375 \mathrm{mg} / \mathrm{m}^{2}$, which were administered intravenously (i.v.). The dose intensity was $100 \%$, regardless of patient blood count.

PET/CT scanning. The Discovery ST16 PET/CT system (GE Medical Systems, Powell, TN, USA) was used to assess ${ }^{18} \mathrm{~F}-\mathrm{FDG}$ distribution in all patients in $3 \mathrm{D}$ mode in a 128x128 matrix. Reconstruction was performed using the 3D 
A

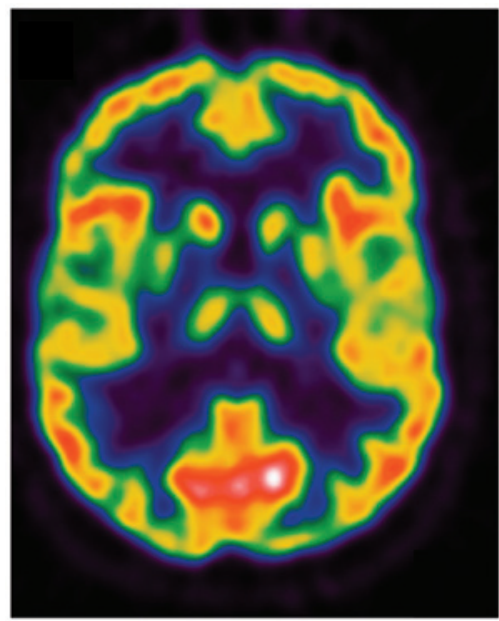

B

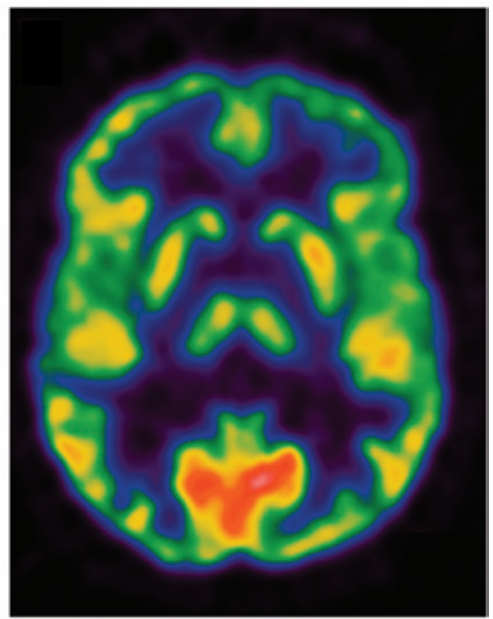

Figure 1. Brain 2-[18F] fluoro-2-deoxy-D-glucose positron emission tomography (PET)/computed tomography scan (A) within one week of Hodgkin disease diagnosis (PET0) and (B) 15 \pm 5 days after the first two adriamycin, bleomycin, vinblastine and dacarbazine cycles (PET2).

A

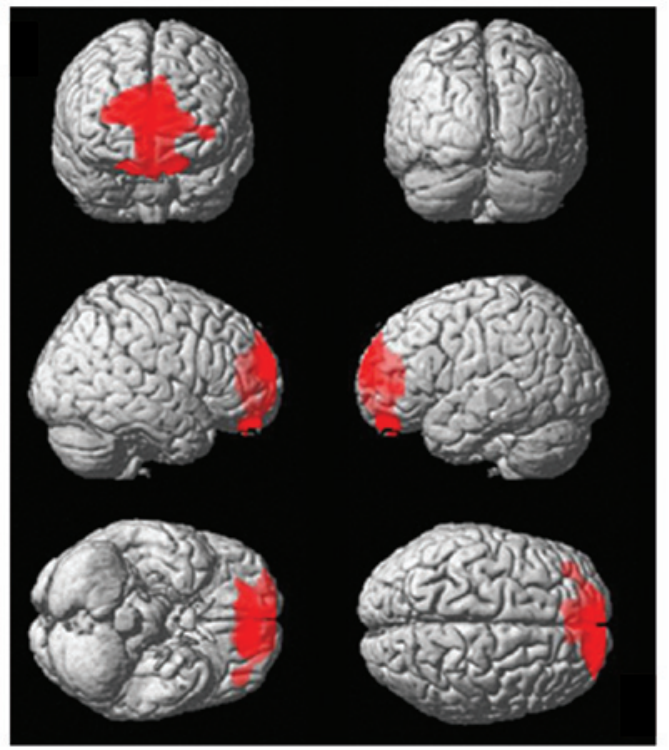

B

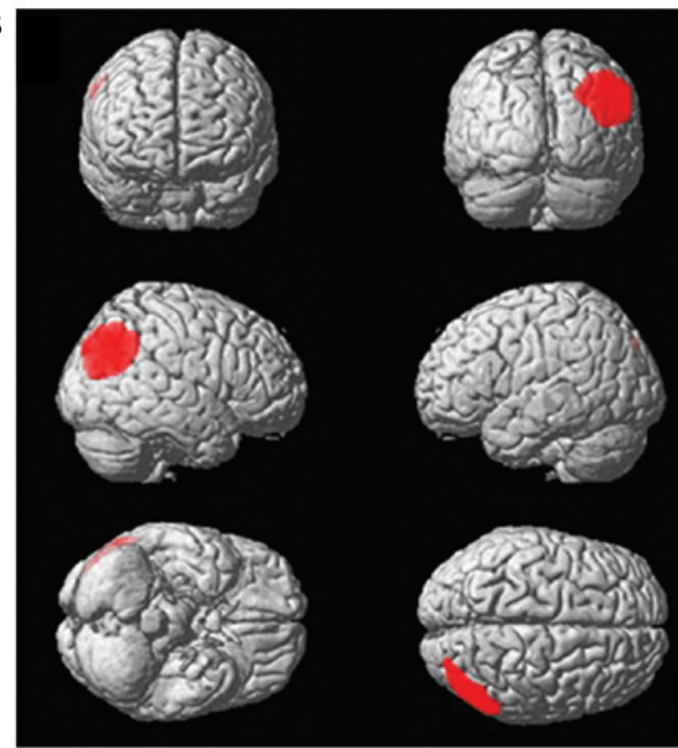

Figure 2. (A) Significant cortical hypometabolism in positron emission tomography (PET)2 when compared with the PET0 group. Threshold of P $<0.05$, corrected for multiple comparisons with false discovery rate at voxel level. (B) High 2-[18F] fluoro-2-deoxy-D-glucose uptake in the right superior parietal lobule in PET2 compared with PET0. Threshold of P $<0.001$, not corrected for multiple comparisons at voxel level. Coordinate and regional details are presented in Table I. PET0, brain 2-[18F] fluoro-2-deoxy-D-glucose $\left({ }^{18} \mathrm{~F}-\mathrm{FDG}\right)$ PET/computed tomography (CT) scan within one week of Hodgkin disease diagnosis; PET2, brain ${ }^{18} \mathrm{~F}-\mathrm{FDG}$ PET/CT scan $15 \pm 5$ days after the first two adriamycin, bleomycin, vinblastine and dacarbazine cycles.

reconstruction method of ordered subsets expectation maximization with 21 subsets and four iterations. The system combines a high-speed ultra 16-detector-row (912 detectors/row) CT unit and a PET scanner with 10,080 bismuth germanate crystals in 24 rings. The axial full width half maximum (FWHM) was $1 \mathrm{~cm}$ (3D mode radius, $5.2 \mathrm{~mm}$ ) and the axial field-of-view was $157 \mathrm{~mm}$. All patients and CG subjects fasted for a minimum of $5 \mathrm{~h}$ prior to i.v. ${ }^{18} \mathrm{~F}-\mathrm{FDG}$ injection to produce a serum glucose level of $\leq 120 \mathrm{mg} / \mathrm{ml}$. All of the subjects in the present study were intravenously injected with $3 \mathrm{MBq} / \mathrm{kg}$ (range, $210-350 \mathrm{MBq}$ ) ${ }^{18} \mathrm{~F}-\mathrm{FDG}$ and hydrated with $500 \mathrm{ml}$ i.v. saline $\mathrm{NaCl} 0.9 \%$.

${ }^{18} \mathrm{~F}-\mathrm{FDG}$ was administered to each patient in a dedicated, dark room. All of the patients were required to remain in a resting condition with closed eyes prior to the PET/CT scan. Following a 45 min rest period, the brain PET/CT scan was performed by placing the patient's head in a support. A low-amperage CT scan of the head was performed for attenuation correction $(40 \mathrm{~mA} ; 120 \mathrm{Kv})$, prior to obtaining the PET image. The duration of the brain PET image set acquisition was $15 \mathrm{~min}$ in all of the patients. All brain PET scans were performed prior to the whole body PET scan, consisting of a low-amperage CT scan for attenuation correction of PET images $(80 \mathrm{~mA} ; 140 \mathrm{kV}$; field of view $\sim 450 \pm 5 \mathrm{~mm}$; CT slice thickness, $3.75 \mathrm{~mm}$ ). The CT Dose Index for ldCT was $4.0175( \pm 0.84) \mathrm{mGy}$ and the dose-length product was $473.296( \pm 161.09) \mathrm{mGy}-\mathrm{cm}$. After non-enhanced CT, totalbody PET examination in the caudocranial direction from the upper thighs to the vertex was performed (every bed of the PET acquisition lasted $3.5 \mathrm{~min}$ ). Reconstruction was performed using the 3 -dimensional reconstruction method 
of ordered-subsets expectation maximization (OSEM) with 30 subsets and two iterations.

Statistical analysis. Differences in brain ${ }^{18} \mathrm{~F}-\mathrm{FDG}$ uptake were analyzed using statistical parametric mapping (SPM2; Wellcome Department of Cognitive Neurology, London, UK) implemented in MATLAB 6.5 (Mathworks, Inc., Natick, MA, USA). PET data were subjected to linear (affine) and non-linear spatial normalization into the Montreal Neurological Institute space. The spatially normalized images were smoothed with a $12-\mathrm{mm}$ isotropic Gaussian filter to blur for individual variations in gyral anatomy and to increase the signal-to-noise ratio. Images were globally normalized to $50 \mathrm{ml} / 100 \mathrm{ml} / \mathrm{min}$ using proportional scaling to remove the confounding effects of changes in the global cerebral glucose consumption, with a masking threshold of 0.8 . The resulting statistical parametric maps, $\operatorname{SPM}\{\mathrm{t}\}$, were transformed into normal distribution (SPM $\{\mathrm{z}\}$ ) units. SPM coordinates were corrected to match the Talairach coordinates, according to the method implemented by Brett (http://imaging. mrc-cbu.cam.ac.uk/imaging/MniTalairach). Subsequently, Brodmann areas (BAs) were identified at a range of 0 to $3 \mathrm{~mm}$ from the corrected Talairach coordinates of the SPM output isocenters, using Talairach Client software (http://www.talairach.org/client.html). Consistent with Bennett et al (15), the threshold of the SPM $\{t\}$ maps was $\mathrm{P}<0.05$, corrected for multiple comparisons using false discovery rate (FDR) at voxel level, and $\mathrm{P}<0.01$, corrected for multiple comparisons using FDR at cluster level. Due to the explorative nature of the present study, when statistically significant differences were not identified at such conservative thresholds, a threshold of $\mathrm{P}<0.001$ uncorrected at voxel level was set. Only those clusters containing $>125$ contiguous voxels (i.e. $>5 \times 5 \times 5$ voxels or $>11 \times 11 \times 11 \mathrm{~mm}$ ) were accepted as significant, due to the partial volume effect caused by the poor spatial resolution of the PET camera $(\sim 2 \times \mathrm{FWHM})$. The voxel-based analyses were performed using a modality-adjusted paired t-test (two conditions, one scan/condition) and the following comparisons were assessed: i) PET0 vs. PET2 and vice versa; ii) PET0 vs. PET6 and vice versa, CG vs. PET0, CG vs. PET2, CG vs. PET6 and vice versa. Age and gender were used as nuisance variables in all analyses and disease staging was added as nuisance variable in the CG vs. PET2 comparison. Two-way analysis of variance was used in demographic data analyses to assess differences in gender and age. $\mathrm{P} \leq 0.05$ was considered to indicate a statistically significant difference and, thus, a valid hypothesis.

\section{Results}

No significant differences were identified in age and gender between HD and CG subjects. However, when PET2 data were subtracted from PET0 and CG data, a significant hypometabolic area including a portion of the orbitofrontal cortex (OFC) bilaterally (BA11) and left anterior cingulate cortex (ACC; BA32; Table I) was identified. When compared with PET0 (Table II; Fig. 1) and CG (Fig. 2) scans, PET2 scans demonstrated a significantly higher ${ }^{18} \mathrm{~F}-\mathrm{FDG}$ uptake distribution in the right angular gyrus (BA39; Table I). No significant differences were identified when subtracting PET6 from PET0 and CG scans and vice versa. Furthermore, the ${ }^{18} \mathrm{~F}$-FDG uptake distribution changes identified at PET2 disappeared at PET6, and no significant changes were identified between PET6 and PET2, PET0 or CG at any of the explored statistical thresholds.

When using the stage of the disease as the nuisance variable, no significant differences were identified in the comparisons between PET0, PET2 or PET6 data and CG data.

\section{Discussion}

The predominant finding of the present study was a significant hypometabolism in OFC bilaterally (BA11) and in the left ACC (BA32), and an increased glucose consumption in the right parietal cortex, in HD patients following the first two CHT cycles. These changes disappeared at the termination of the therapy, six months after diagnosis.

The present study confirms, in a larger series of patients, the results of a previous report and, thus, reinforces its reliability (5). Furthermore, the present study compared the metabolic status of HD patients with that of a CG, improving the design and the overall statistical power of the study.

Possible CHT-induced damage in BAs 11 and 32 may be important in the reduced brain glucose metabolism in these areas following the first two cycles of CHT. Although chemotherapeutic agents typically have restricted direct access to brain tissue due to the blood-brain barrier, animal studies indicate that even chemotherapeutic agents not known to readily cross the blood-brain barrier (for example, doxorubicin used in the present study) are associated with reduced neurogenesis $(16,17)$. In contrast to the previous study using significantly fewer patients (5), the current study did not demonstrate any differences between the PET6 and PET0 ${ }^{18}$ F-FDG distributions. Furthermore, no significant differences were detected between the CG scan and the brain PET scan at the termination of therapy (PET6).

In the case of CHT-induced brain damage, a diffuse cortical and sub-cortical reduction in brain glucose consumption at PET2, followed by a further reduction after four more CHT cycles, would be expected (18). The present study demonstrated that the metabolic changes that occurred during the disease course and the restoration of normal metabolism in the ACC and PFC at the termination of therapy do not appear to be associated with the total dose of CHT administered.

These findings, in particular those derived from the comparison with CG subjects, are in disagreement with a previously published study exploring functional changes in the brains of CHT-treated patients (19), and dispute claims that ABVD CHT may induce permanent brain damage (19). A previous study evaluating the impact of CHT in female breast cancer patients using magnetic resonance imaging indicated that CHT may cause permanent disruption in the networks of various brain regions (in particular in the hippocampus), thus, directly affecting cerebral function in these areas (20). These disruptions may be associated with specific activation/deactivation patterns in different areas of the cerebral cortical during tasks and at rest (for example, the frontal cortex), as reported by Silverman et al (21).

However, a recent study outlined that, during neuropsychological testing, patients who were treated with CHT performed worse than non-cancer control participants. However, CHT-treated patients improved upon their own pre-CHT baseline and performed better than patients treated without CHT, indicating that the majority of long-term cognitive deficits 
associated with CHT-treated breast cancer patients are generally small in magnitude (22).

The lack of a permanent brain metabolic dysfunction identified in the present study may be due to the different types of CHT used and the different periods of examination during the disease course $(\sim 5-10$ years in the cited studies; in the acute phase of CHT in the present case) $(20,21)$. ABVD is considered to be less toxic when compared with other CHT schemes $(3,4)$; however, further studies are required to investigate a possible delayed effect of ABVD CHT on brain function and metabolism.

Various changes in the structure and function of the OFC and ACC have been described in different psychiatric conditions. In particular, structural neuroimaging studies have observed a reduction in the gray matter volume of ACC, and left and right OFC among patients with major depressive disorders $(23,24)$. During traumatic exposure in patients exhibiting post-traumatic stress disorder (PTSD), a significant decrease in cerebral blood flow (CBF) has been identified in the ACC and $\mathrm{OFC}$, and a significant increase in $\mathrm{CBF}$ has been identified in right parietal regions (25-28). The temporary reduced brain glucose metabolism in the OFC and ACC, and the increased metabolism in the right parietal cortex may be explained by the presence of an acute anxiety status in the present patients. This hypothesis is consistent with the development of depressive symptoms in cancer patients caused by the stress of diagnosis with a life-threatening disease (29). Additionally, cancer diagnosis and treatment are accompanied by a number of acute and chronic stressors that can impact the patient quality of life (29).

In the present study, all patients examined by PET6 were disease-free for a minimum of 12 months and presented a negative whole body PET scan after two ABVD cycles. Thus, treatment with two cycles of ABVD is associated with a high disease-free survival rate in HD patients (10-12). It is possible that the good prognosis in the present series of patients resulted in the disappearance of psychiatric symptoms (time period, 6-12 months), as previously reported in patients affected by cancer (30). However, various functional imaging analyses of patients exhibiting mood disorders identified an increased or a decreased CBF or metabolism in BA 11 and 32, despite the majority of patients exhibiting increased CBF or metabolism at rest $(28,31)$.

The largest regional metabolic differences were identified in the comparison of PET2 with PET0 and CG (Tables I and II). At this point of the disease course $(15 \pm 5$ days after the first two ABVD cycles), a high level of anxiety would be expected, due to: (i) The recent diagnosis (within two months) and (ii) the uncertainty of the therapeutic outcome $(12,13)$. This acute anxiety may impact brain metabolism and blood flow. Although the patient may remain in a state of anxiety associated with the possibility of disease recurrence prior to PET6 examination (13), no significant differences in brain metabolism were identified when comparing PET6 with PET0 and CG scans. This supports the hypothesis of an acute and transient psychiatric condition that affects patients early during the disease course.

The severity of the disease appears to be associated with metabolic changes observed in the brains of HD patients. When the disease stage was used as a nuisance variable in the comparison of PET2 and CG scans, no significant difference was identified, possibly due to the patient's perception of a more advanced disease stage as a worse prognostic factor. This is supported by a study performed on a large cohort of patients exhibiting different types of cancer; the presence of metastases was associated with an increased number of anxiety symptoms and early disease stage was associated with fewer depressive symptoms $(7,32)$. However, it has been demonstrated that the distribution of ${ }^{18} \mathrm{~F}$-FDG uptake in healthy tissues is associated with disease progression in HD (33). Furthermore, various studies have demonstrated that the tumor or treatment-induced inflammation can promote the production of peripheral proinflammatory cytokines. Proinflammatory cytokines activate central nervous system pathways that may induce behavioral and affective symptoms, such as a depressed mood, fatigue, anorexia, impaired concentration, sleep disturbance, enhanced pain sensitivity and reduced activity (29,34-36). Therefore, subsequent studies should include longitudinal neuropsychological, psychiatric and laboratory assessments.

In contrast to previous reports (5), the present study demonstrated no significant metabolic changes in BA 10 (prefrontal cortex) in HD patients receiving ABVD CHT. This discrepancy may explained by the larger number of patients examined in the present study, introducing a higher inter-individual variability and, thus, no significant differences. The previous (5) and present cluster of voxels resulting from the group comparisons were almost superimposable and the reported results predominantly differ in the coordinates of the isocenters determined by SPM. However, in respect to its functional role in major depression and/or PTSD, the prefrontal cortex and orbito-frontal cortex voxel clusters superimpose well (28). Furthermore, stress symptoms in cancer patients have been previously described $(30,37,38)$, and several studies describe diagnosis-associated psychological trauma in cancer patients and survivors, for example acute and chronic PTSD $(39,40)$.

As the design of the present study did not consider the co-occurrence of HD and other psychiatric disorders, the possible occurrence of an acute and transient anxiety status is only speculative. The present study achieved its aim of evaluating alterations in brain metabolism in HD patients during various stages of ABVD CHT treatment and compared with a CG; however, the impact of the psychological status of the patient was only considered post hoc. Thus, the lack of a neuropsychological or psychiatric evaluation following disease diagnosis and during treatment is an evident limitation of the present study.

Another possible limitation of the present study is the use of a CG of subjects with a negative brain PET scan, as opposed to a group of healthy volunteers. However, it is important to note that the CG was specifically set up for the present study, and identical protocol and scanners were used for patients and controls. This is important in neuroimaging studies where the number of potential confounding variables must be minimized. The use of a CG comprising of a sample of individuals with a negative PET is common, due to the high costs associated with obtaining a cohort of volunteers. Furthermore, the stringent exclusion criteria were the same as those applied in previous investigations $(41,42)$. In addition, using a CG comprised of neurologically healthy subjects undergoing PET scans for other reasons prevents healthy individuals being exposed to radiations and makes the protocol feasible for the majority of PET centers, as it reduces the costs and efforts required to build up CGs comprised of healthy subjects (43). 
In conclusion, brain metabolic changes in a large cohort of HD patients during the initial phases of CHT are transient and may be due to an acute anxiety state following disease diagnosis and treatment outcome uncertainty. Further studies integrating this data with neuropsychological, psychiatric and laboratory assessments are necessary to confirm the hypotheses of the present study.

\section{References}

1. National Cancer Institute: Cancer statistics: SEER stat fact sheets, Hodgkin lymphoma. http://seer.cancer. gov/statfacts/html/hodg.html. Accessed October, 2013.

2. Bonadonna G, Zucali R, Monfardini S, et al: Combination chemotherapy of Hodgkin's disease with adriamycin, bleomycin, vinblastine and imidazole carboxamide versus MOPP. Cancer 36: 252-259, 1975.

3. Canellos GP and Niedzwiecki D: Long-term follow-up of Hodgkin's disease trial. N Engl J Med 346: 1417-1418, 2002.

4. Borchmann P and Engert A: The past: what we have learned in the last decade. Hematology Am Soc Hematol Educ Program 2010: 101-107, 2010.

5. Chiaravalloti A, Pagani M, Di Pietro B, et al: Is cerebral glucose metabolism affected by chemotherapy in patients with Hodgkin's lymphoma? Nucl Med Commun 34: 57-63, 2012.

6. Pasquini $\mathrm{M}$ and Biondi $\mathrm{M}$ : Depression in cancer patients: a critical review. Clin Pract Epidemiol Ment Health 3: 2, 2007.

7. Spiegel D: Health care. Psychosocial support for patients with cancer. Cancer 74 (Suppl 4): 1453-1457, 1994.

8. Ospedale Santa Croce-Carle Cuneo: Positron Emission Tomography (PET) - Adapted Chemotherapy In Advanced Hodgkin Lymphoma (HL) (HD0607). NLM identifier: NCT00795613. http://clinicaltrials.gov/show/NCT00795613. Accessed October 2010.

9. Varrone A, Asenbaum S, Vander Borght T, et al; European Association of Nuclear Medicine Neuroimaging Committee: EANM procedure guidelines for PET brain imaging using [18F]FDG, version 2. Eur J Nucl Med Mol Imaging 36: 2103-2110, 2009.

10. Lister TA, Crowther D, Sutcliffe SB, et al: Report of a committee convened to discuss the evaluation and staging of patients with Hodgkin's disease: Cotswolds meeting. J Clin Oncol 7: 1630-1636, 1989.

11. Gallamini A, Fiore F, Sorasio R and Meignan M: Interim positron emission tomography scan in Hodgkin lymphoma: definitions, interpretation rules, and clinical validation. Leuk Lymphoma 50: 1761-1764, 2009.

12. Gallamini A, Hutchings M, Rigacci L, et al: Early interim $2-\left[{ }^{18} \mathrm{~F}\right]$ fluoro-2-deoxy-D-glucose positron emission tomography is prognostically superior to international prognostic score in advanced-stage Hodgkin's lymphoma: a report from a joint Italian-Danish study. J Clin Oncol 25: 3746-3752, 2007.

13. Gallamini A, Rigacci L, Merli F, et al: The predictive value of positron emission tomography scanning performed after two courses of standard therapy on treatment outcome in advanced stage Hodgkin's disease. Haematologica 91: 475-481, 2006.

14. Cistaro A, Valentini MC, Chiò A, et al: Brain hypermetabolism in amyotrophic lateral sclerosis: a FDG PET study in ALS of spinal and bulbar onset. Eur J Nuc Med Mol Imaging 39: 251-259, 2011

15. Bennett CM, Wolford GL and Miller MB: The principled control of false 483 positives in neuroimaging. Soc Cogn Affect Neurosci 4: 417-422, 2009.

16. Janelsins MC, Roscoe JA, Berg MJ, et al: IGF-1 partially restores chemotherapy-induced reductions in neural cell proliferation in adult C57BL/6 mice. Cancer Invest 28: 544-553, 2010.

17. Lopes MA, Meisel A, Dirnagl U, et al: Doxorubicin induces biphasic neurotoxicity to rat cortical neurons. Neurotoxicology 29 286-293, 2008

18. Attwell D and Laughlin SB: An energy budget for signaling in the grey matter of the brain. J Cereb Blood Flow Metab 21: 1133-1145, 2001.

19. Zimmer P, Mierau A, Bloch W, et al: Post-chemotherapy cognitive impairment in patients with B-cell non-Hodgkin lymphoma: a first comprehensive approach to determine cognitive impairments after treatment with rituximab, cyclophosphamide, doxorubicin, vincristine and prednisone or rituximab and bendamustine. Leuk Lymphoma: 1-6, 2014.

20. Bruno J, Hosseini SM and Kesler S: Altered resting state functional brain network topology in chemotherapy-treated breast cancer survivors. Neurobiol Dis 48: 329-338, 2012.
21. Silverman DH, Dy CJ, Castellon SA, et al: Altered frontocortical, cerebellar, and basal ganglia activity in adjuvant-treated breast cancer survivors 5-10 years after chemotherapy. Breast Cancer Res Treat 103: 303-311, 2007.

22. Barton MK: Cognitive deficits are usually mild in patients with breast cancer after chemotherapy. CA Cancer J Clin 63: 3-4, 2013.

23. Hakamata Y, Matsuoka Y, Inagaki M, et al: Structure of orbitofrontal cortex and its longitudinal course in cancer-related post-traumatic stress disorder. Neurosci Res 59: 383-389, 2007.

24. Niida A, Niida R, Matsuda H, Inada T, Motomura M and Uechi A: Identification of atrophy of the subgenual anterior cingulate cortex, in particular the subcallosal area, as an effective auxiliary means of diagnosis for major depressive disorder. Int J Gen Med 5: 667-674, 2012.

25. Bonne O, Gilboa A, Louzoun Y, et al: Resting regional cerebral perfusion in recent posttraumatic stress disorder. Biol Psychiatry 54: 1077-1086, 2003.

26. Sachinvala N, Kling A, Suffin S, et al: Increased regional cerebral perfusion by $99 \mathrm{mTc}$ hexamethyl propylene amine oxime single photon emission computed tomography in post-traumatic stress disorder. Mil Med 165: 473-479, 2000.

27. Pagani M, Högberg G, Salmaso D, et al: Regional cerebral blood flow during auditory recall in 47 subjects exposed to assaultive and non-assaultve trauma and developing or not posttraumatic stress disorder. Eur Arch Psychiatry Clin Neurosci 255: 359-365, 2005.

28. Francati V, Vermetten E and Bremner JD: Functional neuroimaging studies in posttraumatic stress disorder: review of current methods and findings. Depress Anxiety 24: 202-218, 2007.

29. Costanzo ES, Sood AK and Lutgendorf SK: Biobehavioral influences on cancer progression. Immunol Allery Clin North Am 31: 109-132, 2011.

30. Mehnert A and Koch U: Prevalence of acute and post-traumatic stress disorder and comorbid mental disorders in breast cancer patients during primary cancer care: a prospective study. Psychooncology 16: 181-188, 2007.

31. Savitz J and Drevets WC: Bipolar and major depressive disorder: neuroimaging the developmental-degenerative divide. Neurosci Biobehav Rev 33: 699-771, 2009.

32. Vodemaier A, Linden W, MacKenzie R, Greig D and Marshall C: Disease stage predicts post-diagnosis anxiety and depression only in some types of cancer. Br J Cancer 105: 1814-1817, 2011.

33. Chiaravalloti A, Danieli R, Abbatiello P, et al: Factors affecting intrapatient liver and mediastinal blood pool ${ }^{18} \mathrm{~F}-\mathrm{FDG}$ standardized uptake value changes during ABVD chemotherapy in Hodgkin's lymphoma. Eur J Nucl Med Mol Imaging 41: 1123-1132, 2014.

34. Wenrib AZ, Sephton SE, Degeest K, et al: Diurnal cortisol dysregulation, functional disability, and depression in women with ovarian cancer. Cancer 116: 4410-4419, 2010.

35. Rich T, Innominato PF, Boerner J, et al: Elevated serum cytokines correlated with altered behavior, serum cortisol rhythm and dampene 24-hour rest-activity patterns in patients with metastatic colorectal cancer. Clin Cancer Res 11: 1757-1764, 2005.

36. Lutgendorf SK, Weinrib AZ, Penedo F, et al: Interleukin-6, cortisol and depressive symptoms on ovarian cancer patients. J Clin Oncol 26: 4820-4827, 2008.

37. Andersen BL, Kiecolt-Glaser JK and Glaser R: A biobehavioral model of cancer stress and disease course. Am Psychol 49: 389-404, 1994

38. Butler LD, Koopman C, Classen C and Spiegel D: Traumatic stress, life events, and emotional support in women with metastatic breast cancer: cancer-related traumatic stress symptoms associated with past and current stressors. Health Psychol 18: 555-560, 1999.

39. Cordova MJ, Studts JL, Hann DM, Jacobsen PB and Andrykowski MA: Symptom structure of PTSD following breast cancer. J Trauma Stress 13: 301-319, 2000.

40. DuHamel KN, Ostrof J, Ashman T, et al: Construct validity of the posttraumatic stress disorder checklist in cancer survivors: analyses based on two samples. Psychol Assess 16: 255-266, 2004.

41. Pagani M, Dessi B, Morbelli S, et al: MCI patients declining and not-declining at mid-term follow-up: FDG-PET findings. Curr Alzheimer Res 7: 287-294, 2010.

42. Nobili F, Mazzei D, Dessi B, et al: Unawareness of memory deficit in amnestic MCI: FDG-PET findings. J Alzheimers Dis 22: 993-1003, 2012.

43. Del Sole A, Clerici F, Chiti A, et al: Individual cerebral metabolic deficits in Alzheimer's disease and amnestic mild cognitive impairment: an FDG PET study. Eur J Nucl Med Mol Imaging 35: 1357-1366, 2008. 